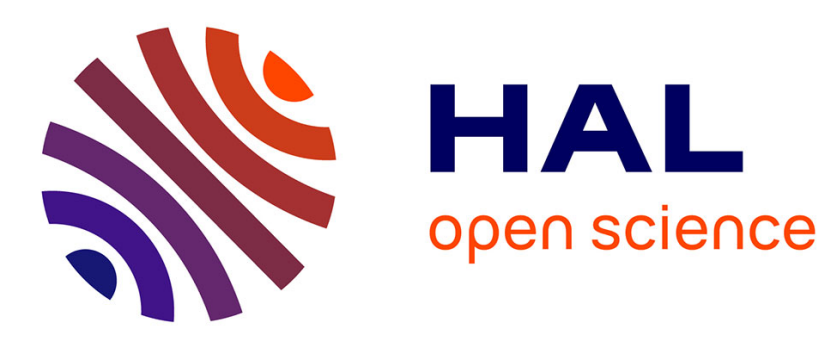

\title{
Fluctuations in 2D defect melting
}

\author{
S. Ami, H. Kleinert
}

\section{To cite this version:}

S. Ami, H. Kleinert. Fluctuations in 2D defect melting. Journal de Physique Lettres, 1984, 45 (18), pp.877-886. 10.1051/jphyslet:019840045018087700 . jpa-00232425

\section{HAL Id: jpa-00232425 https://hal.science/jpa-00232425}

Submitted on 1 Jan 1984

HAL is a multi-disciplinary open access archive for the deposit and dissemination of scientific research documents, whether they are published or not. The documents may come from teaching and research institutions in France or abroad, or from public or private research centers.
L'archive ouverte pluridisciplinaire HAL, est destinée au dépôt et à la diffusion de documents scientifiques de niveau recherche, publiés ou non, émanant des établissements d'enseignement et de recherche français ou étrangers, des laboratoires publics ou privés. 


\title{
LE JOURNAL DE PHYSIQUE-LETTRES
}

Classification

Physics Abstracts

$61.70-64.70$

\section{Fluctuations in 2D defect melting}

\author{
S. Ami $(*)$ and H. Kleinert \\ Freie Universität Berlin, Institut für Theorie der Elementarteilchen, Arnimallee 14, \\ 1000 Berlin 33, F.R.G.
}

(Reçu le 14 mai 1984, accepté le 16 juillet 1984)

\begin{abstract}
Résumé. - Nous étudions la transition de fusion d'un ensemble de défauts en dimension deux. Notre point de départ est un modèle sur réseau, développé récemment, qui représente ces défauts d'une manière duale. Nous calculons d'abord l'approximation du champ moyen, puis les corrections à une boucle et nous donnons le développement de haute température jusqu'à l'ordre $\beta^{11}$. Les résultats sont bien en accord avec des simulations numériques récentes utilisant la méthode de Monte Carlo.

Abstract. - We investigate the melting transition of an ensemble of two-dimensional crystal defects in a recently developed local lattice model, which describes these defects via a duality transformation. We calculate the mean field approximation, the one-loop correction to it, and give the high temperature series up to $\beta^{11}$. The result is in excellent agreement with recent Monte Carlo simulations.
\end{abstract}

\section{Introduction.}

The success in understanding the superfluid phase transitions in two dimensions as an unbinding of vortex pairs [1] or, in three dimensions, as a proliferation of vortex lines [2] has stimulated the investigation of the more complicated process of crystal melting in terms of defects. Topologically, the crystalline analogue of vortices are dislocations [3] with Biot-Savart type long-range forces and a great deal of effort has been spent trying to understand their statistical properties [4-6].

In doing so, however, a simple imitation of the theoretical methods developed for the superfluid turned out to be unable to describe actual physical situation. The basic reason lies in the fact that these methods were devised for a continuous transition, and thus do not apply to melting. In particular, the dilute gas assumption for the defects breaks down during the melting process and, in the molten state, the dislocations reveal a composite nature. In the crystalline state they

(*) Supported by Deutsche Forschungsgemeinschaft under grant K1 256/10-1. 
are tightly bound pairs of disclinations and anti-disclinations. During the melting process, the crystalline forces are screened and this can trigger the dissociations of the bound states making melting a first order transition [7, 8].

There now exists a simple model [9] which describes a gas of disclinations which in the crystalline state are tightly bound to dislocations and, in addition, are practically frozen out up to the extreme vicinity of the melting temperature. It is a straightforward generalization of the $X Y$ spin model of the superfluid. Let us recall that the $X Y$ model is given by a partition function

$$
Z=\prod_{\mathbf{x}} \int_{-\pi}^{\pi} \frac{\mathrm{d} \gamma(\mathbf{x})}{2 \pi} \mathrm{e}^{\beta \sum_{\mathbf{x}, i} \operatorname{Re} U(\mathbf{x}) U^{+}(\mathbf{x}+\mathbf{i})}
$$

where $\mathbf{x}$ are the sites of a simple cubic lattice, $i$ the $D$ oriented links to the next neighbours, and $U(\mathbf{x})=\mathrm{e}^{i \gamma(\mathbf{x})}$ are pure phases. For low $T=1 / \beta$ the model describes spin waves $\sim \int \mathrm{d}^{D} x \frac{\beta}{2}(\partial \gamma)^{2}$.

The phases are observable only modulo $2 \pi$ and this leads to the existence of topological excitations, the vortices.

In a crystal, there exists a similar situation : for low $T=1 / \beta$ there are phonons with an energy

$$
\int \mathrm{d}^{D} x \mu\left[\frac{1}{4} \sum_{i \neq j}\left(\partial_{i} u_{j}+\partial_{j} u_{i}\right)^{2}+\xi \sum_{i}\left(\partial_{i} u_{i}\right)^{2}+\frac{\lambda}{2 \mu}\left(\sum_{i} \partial_{i} u_{i}\right)^{2}\right] \text {. }
$$

The displacement vectors $u_{i}(\mathbf{x})$ of atoms are observable only modulo multiples of the lattice vectors since after a long time the atoms are completely permuted, due to fluctuations. Hence the rescaled displacements $\gamma_{i}(\mathbf{x})=\frac{2 \pi}{a} u_{i}(\mathbf{x})$ are periodic variables of the same type as $\gamma(\mathbf{x})$ in the $X Y$ model. If we neglect, for simplicity, the Lamé constant $\lambda\left({ }^{1}\right)$, this leads directly to the melting model

$$
\begin{aligned}
Z= & \prod_{\mathbf{x}, i} \int_{-\pi}^{\pi} \frac{\mathrm{d} \gamma_{i}(\mathbf{x})}{2 \pi} \times \\
& \times \exp \left\{\beta \operatorname{Re}\left(\sum_{\mathbf{x}, i<j} U_{i}(\mathbf{x}) U_{j}^{+}(\mathbf{x}+\mathbf{i}) U_{i}^{+}(\mathbf{x}+\mathbf{j}) U_{j}(\mathbf{x})+2 \xi \cdot \sum_{\mathbf{x}, i} U_{i}^{+}(\mathbf{x}) U_{i}(\mathbf{x}+\mathbf{i})\right)\right\},
\end{aligned}
$$

where $U_{i}(\mathbf{x}) \equiv \mathrm{e}^{i \gamma_{i}(\mathbf{x})}, \mathbf{x}$ are the sites and $\mathbf{i}$ the oriented links of the lattice, which we shall take to be a s.c. $\left({ }^{2}\right)$.

This model is the symmetric analogue to lattice gauge model, in which the four $U_{i}(\mathbf{x})$ appear with one dagger at a different position $U_{i}(\mathbf{x}) U_{j}(\mathbf{x}+\mathbf{i}) U_{i}^{+}(\mathbf{x}+\mathbf{j}) U_{j}^{+}(\mathbf{x})$. Diagrammatically, the terms in the melting model can be pictured by a distortion diagram those in the gauge model by a rotation diagram $\left({ }^{3}\right)$.

$\left.{ }^{1}\right)$ The duality transformation shows that the $\lambda$ term simply renormalizes the strength of the long-range forces between defects (see, for example Refs. [4] or [7]). Thus its omission should be irrelevant to the qualitative behaviour of the model. This was confirmed by a few exploratory Monte Carlo runs in connection with the work in Ref. [12].

$\left({ }^{2}\right)$ For a real crystal, one should really use triangular lattices. However, the long-range forces between defects are independent of the lattice structure such that a defect model of melting might as well be studied on a simple cubic lattice, for simplicity. The most important reason, however, is the existence of Monte Carlo data for this lattice [12] which helps us to check the accuracy of our methods.

$\left({ }^{3}\right)$ Notice that the $U_{i}(\mathbf{x})$ 's live on the links $\mathbf{i}$ emerging from $\mathbf{x}$. They are represented by an arrow along $\mathbf{i}$, with the operation « dagger " reversing the direction. 
The model (2) has been shown to describe, after a duality transformation, a gas of fundamental crystalline disclinations [10] which can build up dislocations by pairing a Monte Carlo simulation in three " as well as two dimensions" has confirmed the theoretically predicted first order nature of the transition.

Thus, apart from its simplicity, the model reproduces correctly some essential properties of crystalline melting and well deserves further study.

A mean field analysis was given in the original paper (Ref. [7]) where the model was first proposed. Stimulated by the correct size of the transition entropy found in the three dimensional Monte Carlo [11] (MC) simulation, the model was analysed further for $D=3$ [13]. In the cold phase, a one-loop correction brought the internal energy in agreement with the MC data. In the hot phase, a high temperature expansion up to $\beta^{11}$ gave reasonable agreement except in the close vicinity of the transition.

A similar analysis does not yet exist for two dimensional melting. It is the purpose of this paper to provide such an analysis.

\section{Mean field and one-loop correction.}

For this we insert the identity

$$
\int_{-i \infty}^{i \infty} \frac{\mathrm{d} \alpha^{1}}{-2 \pi i} \int_{-i \infty}^{i \infty} \frac{\mathrm{d} \alpha^{2}}{2 \pi i} \int \mathrm{d} u^{1} \mathrm{~d} u^{2} \mathrm{e}^{\sum_{a=1}^{2} \alpha^{a}\left(u^{a}-U^{a}\right)}=\prod_{a=1}^{2} \int \mathrm{d} u^{a} \delta\left(u^{a}-U^{a}\right)=1
$$

for each component $U_{i}(\mathbf{x})$ into (2). This permits replacing the unimodular variable $U_{i}^{a}=\left(\operatorname{Re} U_{i}\right.$, $\left.\operatorname{Im} U_{i}\right)$ in the exponent by the two real variables $u_{i}^{1}, u_{i}^{2}$. The remaining integrals over $\gamma_{i}(\mathbf{x})$ can now easily be performed giving Bessel functions of imaginary arguments $I_{0}\left(\sqrt{\left(\alpha_{i}^{1}\right)^{2}+\left(\alpha_{i}^{2}\right)^{2}}\right)$. This leads to the representation of the partition function

$$
\begin{aligned}
& Z=\prod_{\mathbf{x}, i} \int_{-i \infty}^{i \infty} \frac{\mathrm{d} \alpha_{i}^{1}(\mathbf{x})}{2 \pi i} \int_{-i \infty}^{i \infty} \frac{\mathrm{d} \alpha_{i}^{2}(\mathbf{x})}{2 \pi i} \int \mathrm{d} u_{i}^{1}(\mathbf{x}) \mathrm{d} u_{i}^{2}(\mathbf{x}) \times \\
& \times \exp \left\{\beta \operatorname{Re}\left(\sum_{\mathbf{x}, i<j} u_{i}(\mathbf{x}) u_{j}^{+}(\mathbf{x}+\mathbf{i}) u_{i}^{+}(\mathbf{x}+\mathbf{j}) u_{j}(\mathbf{x})+2 \xi \sum_{\mathbf{x}, i} u_{i}^{+}(\mathbf{x}) u_{i}(\mathbf{x}+\mathbf{i})\right)-\right. \\
&\left.-\frac{1}{2} \sum_{\mathbf{x}, i}\left(\alpha_{i}^{+}(\mathbf{x}) u_{i}(\mathbf{x})+\text { c.c. }\right)+\sum_{\mathbf{x}, i} \ln I_{0}\left(\left|\alpha_{i}(\mathbf{x})\right|\right)\right\}
\end{aligned}
$$

where we have the complex notation $\alpha_{j}=\alpha_{j}^{1}+i \alpha_{j}^{2}, u_{j}=u_{j}^{1}+i u_{j}^{2}$. The exponent is minimal for the constant real mean fields $u_{i} \equiv u, \alpha_{i} \equiv \alpha$

$$
\begin{gathered}
u=\frac{I_{1}(\alpha)}{I_{0}(\alpha)} \\
4 \beta\left[\frac{D-1}{2} u^{3}+\xi u\right]=\alpha
\end{gathered}
$$

which give the free energy density

$$
-\beta f^{\mathrm{MF}}=D\left(\ln I_{0}(\alpha)-\alpha u\right)+D \beta\left(\frac{D-1}{2} u^{4}+2 \xi u^{2}\right) .
$$

The corresponding values of $\alpha, u, f^{\mathrm{MF}}$ are plotted in figures $1 \mathrm{a}, 1 \mathrm{~b}$ for different values of $\xi$. 

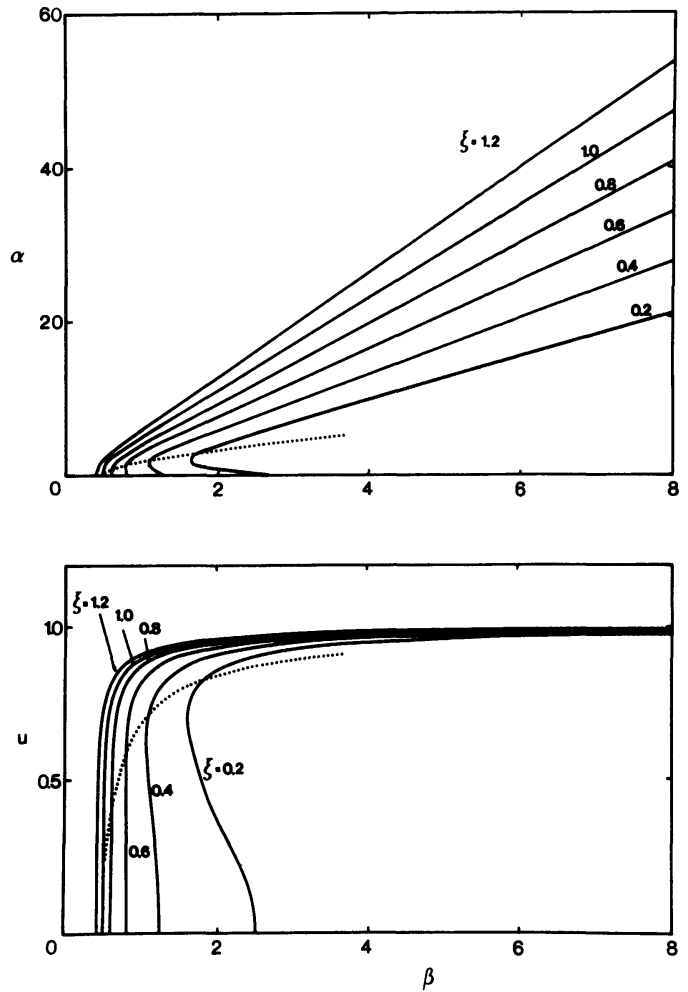

Fig. 1a. - The mean field solutions to the equations (5) of the melting model for different parameters of anisotropy $\xi=\frac{c_{11}-c_{12}}{2 c_{44}}$. The dotted line follows the values of the order parameter right at the melting transition (defined by $-\beta f$ becoming negative).

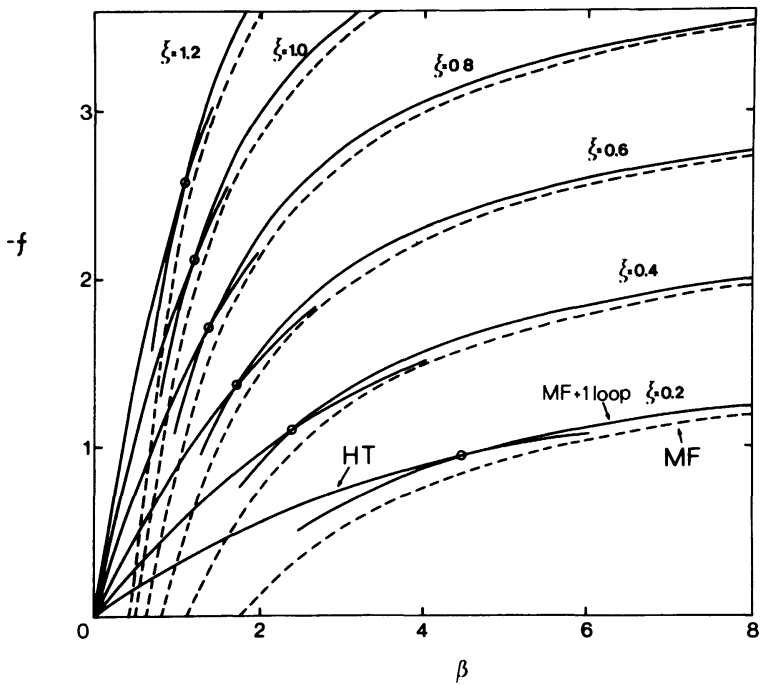

Fig. 1b. - The free energy in mean field approximation (-----), with one loop corrected (-) and the strong coupling approximation up to $\beta^{11}(-)$. The circles mark the transition points. 
Experimentally, $\xi=\frac{c_{11}-c_{12}}{2 c_{44}}$ varies between $0.3(\mathrm{Cu})$ and $1(\mathrm{~W})$. In this approximation the melting transition takes place at

$$
\beta_{\mathrm{m}}^{\mathrm{MF}} \sim \frac{1}{2 \xi^{0.83}}
$$

For $\beta<\beta_{\mathrm{m}}^{\mathrm{MF}}$, the lowest energy is given by $u=\alpha=0,-\beta f^{\mathrm{MF}}=0$.

Let us now calculate the one-loop correction in the crystalline (high $\beta$ ) phase. For this we expand the energy up to quadratic terms and find the exponent

$$
\begin{aligned}
-\beta N f^{\mathrm{MF}}+\beta\left\{\delta ^ { 2 } \operatorname { R e } \left(\sum_{\mathbf{x}, i<j} u_{i}(\mathbf{x}) u_{j}^{+}(\mathbf{x}\right.\right. & \left.\left.+\mathbf{i}) u_{i}^{+}(\mathbf{x}+\mathbf{j}) u_{j}(\mathbf{x})+2 \xi \sum_{\mathbf{x}, i} u_{i}^{+}(\mathbf{x}) u_{i}(\mathbf{x}+\mathbf{i})\right)\right\}- \\
& -\sum_{\mathbf{x}, i}\left(\delta u_{i}^{1}(\mathbf{x}) \delta \alpha_{i}^{1}(\mathbf{x})+\delta u_{i}^{2}(\mathbf{x}) \delta \alpha_{i}^{2}(\mathbf{x})\right)+ \\
& +\sum_{\mathbf{x}, i}\left\{\frac{1}{2} W^{\prime \prime}(\alpha)\left(\delta \alpha_{i}^{1}(\mathbf{x})\right)^{2}+\frac{1}{2 \alpha} W^{\prime}(\alpha)\left(\delta \alpha_{i}^{2}(\mathbf{x})\right)^{2}\right\}
\end{aligned}
$$

where $\delta^{2}$ denotes the quadratic fluctuations of the pure $u$ terms and

$$
\begin{gathered}
W(\alpha)=\ln I_{0}(\alpha), \quad W^{\prime}(\alpha)=\frac{I_{1}(\alpha)}{I_{0}(\alpha)}=u, \\
W^{\prime \prime}(\alpha)=\left(\frac{I_{1}(\alpha)}{I_{0}(\alpha)}\right)^{\prime}=1-\frac{1}{\alpha} \frac{I_{1}(\alpha)}{I_{0}(\alpha)}-\left(\frac{I_{1}(\alpha)}{I_{0}(\alpha)}\right)^{2} \\
=1-\frac{u}{\alpha}-u^{2} .
\end{gathered}
$$

We can now integrate out $\alpha^{1}, \alpha^{2}$ fluctuations which gives

$$
\begin{aligned}
&\left\{\operatorname{det}\left(2 \pi W^{\prime \prime}(\alpha)\right) \operatorname{det}\left(2 \pi \frac{W^{\prime}(\alpha)}{\alpha}\right)\right\}^{-1 / 2} \times \\
& \times \exp \left\{-\frac{1}{2 W^{\prime \prime}(\alpha)} \sum_{\mathbf{x}, i}\left(\delta u_{i}^{1}(\mathbf{x})\right)^{2}-\frac{\alpha}{2 W^{\prime}(\alpha)} \sum_{\mathbf{x}, i}\left(\delta u_{i}^{2}(\mathbf{x})\right)^{2}\right\} .
\end{aligned}
$$

Inserting $u_{i}(\mathbf{x})=u+\delta u_{i}(\mathbf{x})$ into the pure $u$ parts, we calculate its quadratic fluctuation

$$
\begin{aligned}
\frac{1}{2} \delta^{2} & \left\{\sum_{\mathbf{x}, i<j}\left(u_{i}(\mathbf{x}) u_{j}^{+}(\mathbf{x}+\mathbf{i}) u_{i}^{+}(\mathbf{x}+\mathbf{j}) u_{j}(\mathbf{x})+\text { c.c. }\right)+2 \xi \sum_{\mathbf{x}, i}\left(u_{i}^{+}(\mathbf{x}) u_{i}(\mathbf{x}+\mathbf{i})+\mathbf{c . c} .\right)\right\}= \\
= & \frac{u^{2}}{4} \sum_{\mathbf{x}, i \neq j}\left(\delta u_{i}(\mathbf{x}) \delta u_{j}^{+}(\mathbf{x}+\mathbf{i})+\delta u_{i}(\mathbf{x}) \delta u_{i}^{+}(\mathbf{x}+\mathbf{j})+\delta u_{i}(\mathbf{x}) \delta u_{j}(\mathbf{x})+\delta u_{j}^{+}(\mathbf{x}+\mathbf{i}) \delta u_{i}^{+}(\mathbf{x}+\mathbf{j})+\right. \\
& \left.+\delta u_{j}^{+}(\mathbf{x}+\mathbf{i}) \delta u_{j}(\mathbf{x})+\delta u_{i}^{+}(\mathbf{x}+\mathbf{j}) \delta u_{j}(\mathbf{x})+\mathbf{c . c} .\right)+\xi \sum_{\mathbf{x}, i}\left(\delta u_{i}^{+}(\mathbf{x}) \delta u_{i}(\mathbf{x}+\mathbf{i})+\text { c.c. }\right)
\end{aligned}
$$

Using the lattice gradient

$$
\begin{aligned}
& \nabla_{i} u_{j}(\mathbf{x})=u_{j}(\mathbf{x}+\mathbf{i})-u_{j}(\mathbf{x}) \\
& \nabla_{i} u_{i}(\mathbf{x})=u_{j}(\mathbf{x})-u_{j}(\mathbf{x}-\mathbf{i})
\end{aligned}
$$


we can rewrite this as follows

$$
\begin{aligned}
\frac{u^{2}}{4} \sum_{\mathbf{x}, i \neq j}\left(\delta u_{i}(\mathbf{x})\left(1+\nabla_{i}\right) \delta u_{j}^{+}(\mathbf{x})\right. & +\delta u_{i}(\mathbf{x})\left(1+\nabla_{j}\right) \delta u_{i}^{+}(\mathbf{x})+ \\
& +\delta u_{i}(\mathbf{x}) \delta u_{j}(\mathbf{x})+\left(1+\nabla_{i}\right) \delta u_{j}^{+}(\mathbf{x})\left(1+\nabla_{j}\right) \delta u_{i}^{+}(\mathbf{x}) \\
& \left.+\delta u_{j}(\mathbf{x})\left(1+\nabla_{i}\right) \delta u_{j}^{+}(\mathbf{x})+\delta u_{j}(\mathbf{x})\left(1+\nabla_{j}\right) \delta u_{i}^{+}(\mathbf{x})+\text { c.c. }\right) \\
& +\xi \sum_{\mathbf{x}, i}\left(\delta u_{i}^{+}(\mathbf{x})\left(1+\nabla_{i}\right) \delta u_{i}(\mathbf{x})+\text { c.c. }\right)
\end{aligned}
$$

and finally, as

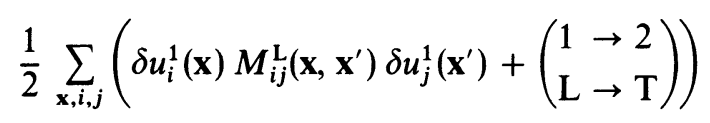

where the matrices $M_{i j}^{\mathbf{L}}$ are given by

$$
\begin{aligned}
& M_{i j}^{\mathrm{T}}\left(\mathbf{x}, \mathbf{x}^{\prime}\right)=u^{2}\left\{2\left(1+\nabla_{i}\right) \pm 1 \pm\left(1-\bar{\nabla}_{j}\right)\left(1+\nabla_{i}\right)+\right. \\
& \left.+2\left[\sum_{l}\left(1+\nabla_{l}\right)-2\left(1+\nabla_{i}\right) \mp 1\right] \delta_{i j}\right\} \delta_{\mathbf{x}, \mathbf{x}^{\prime}}+4 \xi\left(1+\nabla_{i}\right) \delta_{i j} \delta_{\mathbf{x}, \mathbf{x}^{\prime}} .
\end{aligned}
$$

These matrices are diagonal in momentum space

$M_{i j}^{\mathrm{T}}(\mathbf{k})=u^{2}\left\{ \pm\left(\mathrm{e}^{i k_{i}} \pm 1\right)\left(\mathrm{e}^{-i k_{j}} \pm 1\right)+2 \delta_{i j}\left(\sum_{l} \cos k_{l}-2 \cos k_{i} \mp 1\right)\right\}+4 \xi \delta_{i j} \cos k_{i}$

For long wavelengths, the full fluctuation matrices, given by

$$
D_{i j}^{\mathrm{L}}=\frac{1}{W^{\prime \prime}(\alpha)} \delta_{i j}-\beta M_{i j}^{\mathrm{L}}, \quad D_{i j}^{\mathrm{T}}=\frac{\alpha}{W^{\prime}(\alpha)} \delta_{i j}-\beta M_{i j}^{\mathrm{T}}
$$

behave as follows

$$
\begin{aligned}
& D_{i j}^{\mathrm{L}} \simeq \beta\left[\left(\frac{1}{\beta W^{\prime \prime}(\alpha)}+2 u^{2}-4 \xi\right) \delta_{i j}-4 u^{2}+2 i u^{2}\left(-k_{i}+k_{j}\right)+\right. \\
& \left.+u^{2}\left\{\left(k^{2}+2\left(\frac{\xi}{u^{2}}-1\right) k_{i}^{2}\right) \delta_{i j}+k_{i}^{2}+k_{j}^{2}-k_{i} k_{j}\right\}\right] \\
& D_{i j}^{\mathrm{T}} \simeq \beta u^{2}\left\{\left(k^{2}+2\left(\frac{\xi}{u^{2}}-1\right) k_{i}^{2}\right) \delta_{i j}+k_{i} k_{j}\right\}
\end{aligned}
$$

The transverse modes are massless. They are the sound waves in a crystal with temperature softened elastic constants

$$
\begin{gathered}
c_{44}(T)=c_{44} u^{4} \\
\left(c_{11}-c_{12}\right)(T)=\left(c_{11}-c_{12}\right) u^{2} .
\end{gathered}
$$


The quadratic fluctuations give a loop contribution to the free energy density

$$
-\beta f^{1 \text { loop }}=-\frac{1}{2} \int_{-\pi}^{\pi} \frac{\mathrm{d}^{2} k}{(2 \pi)^{2}}\left\{\sum_{i} \ln N_{i}^{\mathrm{L}}+\ln \left(1-\varepsilon^{\mathrm{L}} C^{\mathrm{L}}\right)+(\mathrm{L} \rightarrow \mathrm{T})\right\}
$$

where

$$
\begin{gathered}
\varepsilon^{\mathrm{L}}=\beta u^{2}\left(1-\frac{u}{\alpha}-u^{2}\right), \quad \varepsilon^{\mathrm{T}}=\beta \frac{u^{3}}{\alpha} \\
N_{i}^{\mathrm{T}}=1-2 \varepsilon^{\mathrm{T}}\left\{\sum_{l}^{\mathrm{L}} \cos k_{l}+2\left(\frac{\xi}{u^{2}}-1\right) \cos k_{i} \mp 1\right\} \\
C^{\mathrm{T}}= \pm \sum_{j} \frac{2\left(1 \pm \cos k_{j}\right)}{N_{j}^{\mathrm{T}}} .
\end{gathered}
$$

For large $\beta$, the 1 loop contributes just the black body radiation of the sound waves and gives $-\beta f^{1 \text { loop }} \simeq \ln (2(2 \xi+1) / \sqrt{2 \xi})-$

$$
-\frac{1}{2} \int_{-\pi}^{\pi} \frac{\mathrm{d}^{2} k}{(2 \pi)^{2}} \ln \left\{\left(\sum_{i} 2\left(1-\cos k_{i}\right)\right)^{2}+2(\xi-1) \prod_{i} 2\left(1-\cos k_{i}\right)\right\} .
$$

The integral can be evaluated numerically.

For $\xi=1$ it is 2.34 .

If we take also the mean field solution to the same order in $1 / \beta$ we find

$-\beta f^{\mathrm{MF+1} \text { loop }} \simeq-\ln (2 \pi \beta \sqrt{2 \xi})+(1+4 \xi) \beta-$

$$
-\frac{1}{2} \int_{-\pi}^{\pi} \frac{\mathrm{d}^{2} k}{(2 \pi)^{2}} \ln \left\{\left(\sum_{i} 2\left(1-\cos k_{i}\right)\right)^{2}+2(\xi-1) \prod_{i} 2\left(1-\cos k_{i}\right)\right\} .
$$

For $\xi=1$ this is

$$
\simeq-\ln (2 \sqrt{2} \pi \beta)+5 \beta-1.17 .
$$

The full one loop corrected $-\beta f$ deviates very little from this high $\beta$ limiting form.

For $\beta<\beta_{\mathrm{m}}^{\mathrm{MF}}$, the mean field energy is zero. In order to calculate fluctuation corrections to this, we perform the high temperature expansion,

$$
\begin{aligned}
Z=\prod_{\mathbf{x}, i} \int_{-\pi}^{\pi} \frac{\mathrm{d} \gamma_{i}(\mathbf{x})}{2 \pi} \mathrm{e}^{\beta\left\{\sum_{\mathbf{x}, i<j} \cos \left(\nabla_{i} \gamma_{j}+\nabla_{j} \gamma_{i}\right)+2 \xi \sum_{\mathbf{x}, i} \cos \left(\nabla_{i} \gamma_{i}\right)\right\}=} \\
=\sum_{\left\{\sigma_{i j}(\mathbf{x})\right\}} \prod_{\mathbf{x}, i<j} I_{\sigma_{i j}(\mathbf{x})}(\beta) \prod_{\mathbf{x}, i} I_{\sigma_{i i}(\mathbf{x})}(2 \xi \beta) \times \mathrm{e}^{i} \sum_{\mathbf{x}, i<j} \sigma_{i j}\left(\nabla_{i} \gamma_{j}+\nabla_{j} \gamma_{i}\right)+i \sum_{\mathbf{x}, i} \sigma_{i i} \nabla_{i} \gamma_{i}
\end{aligned}
$$

Integrating out the $\gamma_{i}$ variables gives the stress conservation law

$$
\bar{\nabla}_{j} \sigma_{i j}(\mathbf{x})=0 .
$$

The lowest contribution to the free energy is

$$
-\beta f=\ln I_{0}(\beta)+2 \ln I_{0}(2 \xi \beta) .
$$


The next correction comes from the lowest integer $\sigma_{i j}$ configuration satisfying (20). Writing $\sigma_{i j}(\mathbf{x})=\varepsilon_{i l} \varepsilon_{j m} \bar{\nabla}_{l} \bar{\nabla}_{m} \chi(\mathbf{x})$ with integer $\chi$ and setting $\chi(\mathbf{x})=\delta_{\mathbf{x}, \mathbf{0}}$ we see that this lowest contribution leads to a first correction to (21) of the form $2\left(\frac{I_{1}(\beta)}{I_{0}(\beta)}\right)^{4}\left(\frac{I_{2}(2 \xi \beta)}{I_{0}(2 \xi \beta)}\right)^{2}\left(\frac{I_{1}(2 \xi \beta)}{I_{0}(2 \xi \beta)}\right)^{4}\left({ }^{4}\right)$. This has a threshold behaviour of $\beta^{12}$ and turns out to be numerically negligible up to the melting temperature.

In figures $1 \mathrm{~b}, 2$ we display our free energy, and in figure 2 the internal energy $E=\frac{\partial}{\partial \beta}(\beta f)+5$ as a function of $\beta$ and compare the latter with the Monte Carlo data of reference [12]. The agreement is seen to be excellent. In table I we show the inverse melting temperature $\left({ }^{5}\right)$ as a function of $\xi$ which has the same $\xi$ dependence as (6) but is renormalized by an overall factor $\beta_{\mathrm{m}} \sim 1.2 \xi^{-0.83}$.

It is interesting to find out that the fluctuations play an important role in generating the transition entropy. At the mean field level, the point $\xi=1$ is tricritical. Near it, $-\beta f$ has the Landau expansion

$$
-\beta f^{\mathrm{MF}}=\left(\beta \xi-\frac{1}{2}\right) \alpha^{2}+\frac{1}{2^{5}}(1-\xi) \alpha^{4}-\frac{5}{2^{7} 3^{2}} \alpha^{6}
$$
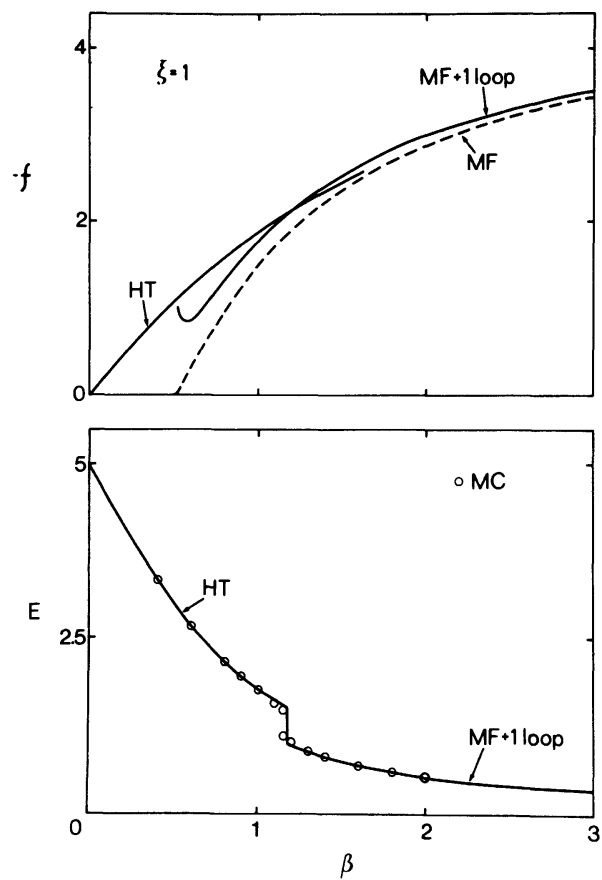

Fig. 2. - The calculated free energy and its derivative, the internal energy $E=\frac{\partial}{\partial \beta}(\beta f)+5$ in comparison with the Monte Carlo data of Ref. [10]. The value of $\xi$ is fixed to unity.

$\left({ }^{4}\right)$ With $\chi(\mathbf{x})=\delta_{\mathbf{x}, 0}$ we have $\sigma_{11}(0)=1, \quad \sigma_{11}(2)=-2, \sigma_{11}(22)=1 \quad \sigma_{22}(0)=1, \sigma_{22}(1)=-2$, $\sigma_{22}(\mathbf{2 1})=-1, \quad \sigma_{12}(0)=\sigma_{21}(0)=-1, \quad \sigma_{12}(1)=\sigma_{21}(1)=1, \quad \sigma_{12}(2)=\sigma_{21}(2)=1, \quad \sigma_{12}(1+2)=$ $=\sigma_{21}(1+2)=-1$, and all others zero.

$\left({ }^{5}\right)$ Measured in units of $\frac{4 \pi^{2} k_{\mathrm{B}}}{\mu a^{2}}$. 
Table I. - The inverse melting temperatures in units of $4 \pi^{2} k_{\mathrm{B}} /\left(\mu a^{2}\right)$ for different parameters of anisotropy $\xi=\left(c_{11}-c_{12}\right) /\left(2 c_{44}\right)$ in various approximate schemes compared with Monte Carlo results in Ref. [10].

\begin{tabular}{|c|c|cc|c|}
\hline$\xi$ & MF & HT, LT & $\beta_{\mathrm{m}}$ HT, MF + 1 loop & Monte Carlo [10] \\
\hline 0.2 & 1.77 & 4.84 & 4.46 & \\
0.4 & 1.12 & 2.70 & 2.38 & $2.35 \pm 0.05$ \\
0.6 & 0.81 & 1.98 & 1.71 & \\
0.8 & 0.62 & 1.61 & 1.38 & $1.36 \pm 0.02$ \\
1.0 & 0.50 & 1.39 & 1.18 & $1.15 \pm 0.01$ \\
1.2 & 0.42 & 1.23 & 1.04 & \\
\hline
\end{tabular}

MF, HT, LT, MF + 1 loop stand for mean field approximation in equations (5), strong coupling approximation up to $\beta^{11}$ in equation (21), low temperature expansion up to $1 / \beta$ in equation (19), and one loop corrected mean field approximation in equations (5), (18), respectively.

and we see that the quartic term turns negative for $\xi>1$ implying a second order transition. This property does not, however, survive the fluctuation corrections, after which the entropy jump remains positive for all physically acceptable values of $\xi$.

If is useful to compare this model with the closely related lattice gauge theory (LGT). The mean field theories and the one-loop corrections are very similar. At this level of approximation, both have a strong first order transition. In the LGT, fluctuations make precisely the opposite way and the transition is removed completely. In a plot like ours this would show up by the high temperature expansion for $-\beta f$ lying always above the mean field plus one loop curve, with no intersection taking place.

\section{Acknowledgments.}

The authors are grateful to W. Janke, T. Hofsäss and T. Matsui for discussions.

\section{References}

[1] BerezinskiI, V. L., Sov. Phys. JETP 32 (1971) 493.

Kosterlitz, J. M. and Thouless, D. J., J. Phys. C 6 (1973) 1181, Prog. Low Temp. Phys. B 7 (1978) 371.

Kosterlitz, J. M., J. Phys. C 7 (1974) 1046.

[2] Kleinert, H., Phys. Lett. A 93 (1982) 86.

[3] Nabarro, F. R. N., Theory of Dislocations (Clarendon Press, Oxford) 1967.

[4] Nelson, D. R. and Halperin, B. I., Phys. Rev. B 19 (1979) 2457.

Young, A. P., Phys. Rev. B 19 (1979) 1855.

ZipPelius, A., HalPerin, B. I. and Nelson, D. R., Phys. Rev. B 22 (1980) 2514 ;

See also the review Brinkman, W. F., Fisher, D. S., Moncton, D. E. in Science 217 (1982) 693.

[5] Nelson, D. R., Phys. Rev. B 26 (1982) 269. 
[6] The most recent Monte Carlo studies on dislocation gases were done in SAIto, Y., Phys. Rev. Lett. 48 (1982) 1114 ; Phys. Rev. B 26 (1982) 6239. This author found empirically that a removal of the core energies led to a first order transition. A similar argument had been put forward in three dimensions on purely theoretical grounds in KLEINERT, H., Lett. Nuovo Cimento 34 (1982) 466, on the basis of the observation that the Ginzburg-Landau theory of defects and stresses should have a first order transition for small core energies of the lines (i.e. for small steric repulsion), the tricritical point being calculated in KLEINERT, H., Lett. Nuovo Cimento 35 (1982) 405.

[7] Kleinert, H., Phys. Lett. A 95 (1983) 381.

[8] Kleinert, H., Phys. Lett. A 95 (1983) 493.

[9] Kleinert, H., Lett. Nuovo Cimento 34 (1982) 464.

[10] Kleinert, H., Phys. Lett. A 91 (1982) 295. See Ref. [5] for a discussion of the ensemble of disclinations. This author concludes that there should be two successive continuous transitions, an expectation which is not borne out by the present analysis and the Monte Carlo simulation in Ref. [12].

[11] JacoBs, L. and Kleinert, H., J. Phys. A 17 (1984) L361.

[12] JANKE, W. and KLEINERT, H., Berlin preprint.

[13] Ami, S., Hofsäss, T. and Horsley, R., Phys. Lett. A 101 (1984) 145. 